\title{
IceCube Search for Neutrinos from 1ES 1959+650: Completing the Picture
}

\author{
The IceCube ${ }^{1}$, FACT $^{2}$ and MAGIC ${ }^{3}$ Collaborations \\ ${ }^{1}$ http://icecube.wisc.edu/collaboration/authors/icrc17_icecube \\ ${ }^{2}$ http://fact-project.org/collaboration/icrc2017_authorlist.html

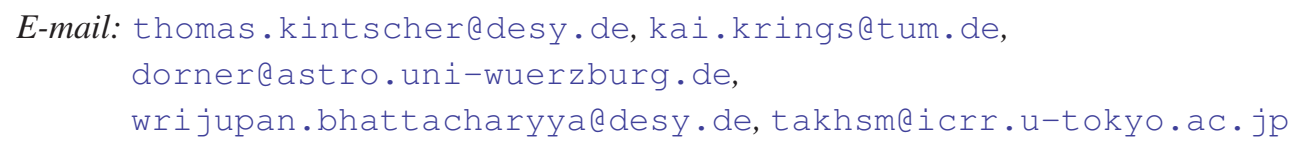

The IceCube neutrino observatory is a $1 \mathrm{~km}^{3}$ detector deployed in the ice at the South Pole. While it has observed an astrophysical flux of neutrinos, individual sources have yet to be identified and the high-peaked BL Lac object 1ES 1959+650 is an intriguing candidate. It exhibited an "orphan flare" in 2002: a TeV gamma ray flare without a simultaneous X-ray flare, behavior that is hard to accommodate in leptonic synchrotron self-compton models. This suggests that it is a potential site of hadronic acceleration and thus a prime source candidate for neutrinos. A recently observed increase in gamma ray activity from 1ES 1959+650 between May and July of 2016 has provided a new opportunity, prompting a dedicated search in IceCube data for neutrinos correlated with the flaring states during this period. We present results based on two model-independent approaches which look only for an excess of neutrinos from the source, as well as a more targeted search for a direct time correlation between neutrino events observed by IceCube and gamma ray emission observed with the FACT and MAGIC telescopes.

Corresponding authors: Thomas Kintscher*1a, Kai Krings ${ }^{1 b}$, Daniela Dorner ${ }^{23 c}$, Wrijupan Bhattacharyya $^{3 \mathrm{a}}$, Mitsunari Takahashi ${ }^{3 \mathrm{~d}}$

${ }^{a}$ Deutsches Elektronen-Synchrotron (DESY), 15738 Zeuthen, Germany

${ }^{b}$ Technische Universität München, 85748 Garching, Germany

${ }^{c}$ Universität Würzburg, 97074 Würzburg, Germany

${ }^{d}$ Institute for Cosmic Ray Research, University of Tokyo, Japan

35th International Cosmic Ray Conference - ICRC2017 -

10-20 July, 2017

Bexco, Busan, Korea

* Speaker. 


\section{Introduction}

Ever since the discovery of cosmic rays more than 100 years ago, the field of astroparticle physics strives to locate and understand their sources. In the pursuit of this goal, this work focuses on two cosmic messengers: gamma rays and neutrinos. The former are subject to intergalactic absorption effects [1], while the latter may also be detected from sources at high redshifts. Both particles' uncharged nature leaves them undeflected by (inter-)galactic magnetic fields, enabling observers to point back at their origin and provide a complementary picture of the sources.

1ES 1959+650, at a redshift of $z=0.047$ [2], belongs to the class of high-frequency peaked BL Lac objects (HBL), i.e. active galactic nuclei with jets pointed towards the observer and a spectral energy distribution characterized by two broad humps in the UV-X-ray and GeV-TeV energy ranges without emission lines. It was established as a very high energy (VHE, $E>100 \mathrm{GeV}$ ) gamma ray emitter [3, 4]. The most notable VHE flaring episode was recorded on June 4th, 2002 by Whipple, when a gamma ray flare without a simultaneous increase in X-ray emission was detected from this source $[3,5,6]$. Several emission models were proposed to describe this "orphan" gamma ray flare. A simple Synchrotron Self Compton (SSC) one [5] was found to underpredict the observed radio and optical fluxes. Alternatively, hadronic models, such as the "mirror model" [7] were also developed for this event and predict a correlated neutrino flux.

A search for neutrino events correlated with the VHE flare was performed on the data from AMANDA $[8,9]$. Three neutrino events were found to arrive during the flaring episode, including one simultaneous with the peak of the "orphan" flare. Yet, no reliable estimate of the significance could be determined as the analysis was not performed in a blind fashion.

Since 2002 1ES1959+650 has remained in a quiescent state in gamma rays [10, 11]. Also, no signal was detected from this source on top of the background in a dedicated search on 7 years of IceCube data [12].

More recently, increased gamma ray activity of 1ES 1959+650 with gamma ray flux levels of the order of the 2002 flare, has been observed between April and July 2016 [13]. This new flare provides another, rare opportunity to investigate a direct connection between the neutrino and VHE gamma ray emission in 1ES 1959+650.

\section{Observatories}

IceCube is a neutrino observatory at the geographic South Pole [14] consisting of over 5000 digital optical modules (DOMs) buried at depths between 1450 and 2450 meters in the Antarctic glacier. Cherenkov radiation from charged particles, produced in interactions of neutrinos in the ice or the nearby bedrock, is detected and used to reconstruct the direction, energy and to some extent the flavor of the neutrinos.

Of particular interest to this analysis are charged-current interactions of muon neutrinos. The resulting muon traverses the ice and its track-like signature allows for a reconstruction of the direction. The source is located in the Northern sky, allowing for an analysis with a selection of upgoing tracks in the detector, which significantly reduces the the background from atmospheric muons.

Several available event selection methods have been compared. Eventually, the one described in [15] was chosen as it provides the best discovery potential at this particular declination. It 
relies on boosted decision trees (BDTs) to suppress the backgrounds from both misreconstructed downgoing muons as well as electron neutrino and neutral-current muon neutrino interactions.

FACT, the First G-APD Cherenkov Telescope, located next to the two MAGIC telescopes at $2200 \mathrm{~m}$ a.s.l. on the Canary Island of La Palma, is equipped with a camera using novel silicon photomultipliers (SiPM; also Geiger-mode Avalanche Photo Diodes, G-APDs) providing an excellent performance [16]. As SiPMs do not degrade when exposed to bright light, the duty cycle of the instrument is maximized and the gaps in the light curves around full moon are minimized. Monitoring blazars at $\mathrm{TeV}$ energies, FACT provides an unprecedented dense and unbiased data sample [17], which is ideally suited to constrain the neutrino flux from gamma ray light curves.

MAGIC is a system of two $17 \mathrm{~m}$ Cherenkov telescopes, combining a large mirror area, allowing for observations of gamma rays with energies as low as $50 \mathrm{GeV}$, with the stereoscopic technique providing strong hadronic background rejection. The integral sensitivity achieved in the energy range $>220 \mathrm{GeV}$ is at the level of $0.66 \%$ of the Crab Nebula flux in $50 \mathrm{~h}$ of observations, when considering point-like sources with Crab Nebula-like spectrum [18]. The angular resolution at those energies is $\leq 0.07^{\circ}$. As detailed in [19], MAGIC can also observe under moonlight conditions. The MAGIC telescopes performed the observations of 1ES 1959+650 in April-July during moonless nights and moon time, with zenith angles in the range of 35 to 52 degrees. The collected data were analyzed in the MARS analysis framework by means of the standard stereoscopic analysis routines [20].

\section{Analysis Methods}

The IceCube analyses are based on an unbinned maximum-likelihood method [21], considering each event's individual properties, such as the direction and its uncertainty, an energy proxy, and the arrival time of the neutrino. The exact formulation of the likelihood varies between the three analyses, and will be described in the following sections.

\subsection{Time-Integrated Likelihood Analysis with IceCube}

This analysis makes the least amount of assumptions about the time structure of a potential signal. As the total time window is short compared to previous IceCube point source searches [12], the formulation of the test statistic $\Lambda$ is modified with respect to the original one from [21] assuming the average background event rate $\left\langle n_{b}\right\rangle$ is known: [22]:

$$
\Lambda\left(n_{s}, \gamma\right)=-n_{s}+\sum_{i}^{\text {events }} \log \left[\frac{n_{s}}{\left\langle n_{b}\right\rangle} \frac{S_{i}(\gamma)}{B_{i}}\right] \quad \text { with } \quad \frac{S_{i}}{B_{i}}=\frac{\mathscr{S}_{\text {sig }}\left(\alpha_{i}, \delta_{i}, \sigma_{i}\right)}{\mathscr{S}_{\mathrm{bkg}}\left(\delta_{i}\right)} \frac{\mathscr{E}_{\mathrm{sig}}\left(E_{i}, \delta_{i} \mid \gamma\right)}{\mathscr{E}_{\mathrm{bkg}}\left(E_{i}, \delta_{i}\right)}
$$

The amount of signal $n_{s}$ and its spectral index $\gamma$ are free parameters in the fit. The probability densities $S_{i}$ and $B_{i}$ for an event to belong to the signal or background, respectively, consider the spatial and energy information, where $\alpha$ and $\delta$ are the reconstructed right ascension and declination, $\sigma$ is the estimated, circularized uncertainty on the direction, and $E$ is a proxy for the muon energy at the detector.

The spatial term $\mathscr{S}_{\text {sig }}$ assumes a two-dimensional Gaussian distribution of the signal with a width of $\sigma$ around the fixed location of the source, compared to a uniform distribution $\mathscr{S}_{\mathrm{bkg}}$ of 
background events. The energy term $\mathscr{E}_{\text {sig }}$ considers the probability for a certain observation $E$ given a spectral index of $\gamma$ of the signal versus the energy spectrum of the atmospheric background $\mathscr{E}_{\mathrm{bkg}}$. In order to minimize the influence of a potential signal, the background event rate $\left\langle n_{b}\right\rangle$ and its energy spectrum are derived using the off-time data recorded between May 2015 and April 2016, i.e. before the flaring activity of the source.

Eventually, the test statistic is maximized with respect to $n_{s}$ and $\gamma$. The spectral index is bound to $\gamma \in[-4,-1]$, and the signal strength must be non-negative $\left(n_{s} \geq 0\right)$. Thus the test statistic can take values of $\Lambda \geq 0$.

The distribution of the test statistic in the absence of a signal is obtained from trials on off-time data, scrambled in right ascension: The arrival times of the neutrinos are shuffled and the transformation from detector coordinates into equatorial coordinates is repeated. While any potential spatial clustering is destroyed, detector effects and correlations between the other variables used in the analysis are retained.

\subsection{Time-Clustering Analysis with IceCube}

In this approach, it is assumed that a potential burst of neutrinos is clustered in time, superimposed on a uniform distribution of background events.

The so-called "time-clustering" algorithm defines subsets of events (clusters) in time and maximizes the likelihood for each subset. Afterwards, the cluster with the highest test statistic is chosen and its significance is evaluated.

As the number of possible clusters grows rapidly, it is useful to limit the trials to the promising ones, which contain events close to the source or of very energetic nature. Therefore, the events fulfilling $S_{i} / B_{i}>1$ mark the boundaries of clusters, which are tested using the likelihood formulation from [21]

$$
\mathscr{L}=\prod_{i=1}^{N}\left[\frac{n_{s}}{N} S_{i}+\left(1-\frac{n_{s}}{N}\right) B_{i}\right]
$$

with $N$ being the number of events in the candidate cluster. Comparing the sensitivity of this analysis to that of the aforementioned time-integrated analysis, only time windows no longer than 21 days $\left(T_{\max }\right)$ need to be tested.

The likelihood maximization yields the best fit values of $\hat{n}_{s}$ and $\hat{\gamma}$. More short time windows than long time windows can be defined, and a bias towards short time windows needs to be avoided. A marginalization term [23] in the test statistic corrects for the number of trials with a duration $T_{\text {sig }}$ :

$$
\Lambda=-2 \log \left(\frac{\mathscr{L}\left(\hat{n}_{s}, \hat{\gamma}\right)}{\mathscr{L}(0,-)} \frac{T_{\mathrm{sig}}}{T_{\max }}\right)
$$

As before, the background distribution of the test statistic is derived from trials on scrambled offtime data. An example is shown in Fig. 1.

\subsection{Correlation between Neutrinos and Gamma Rays}

Whereas the previous analyses have focused solely on neutrinos, the third analysis combines the data from the IceCube experiment with the observations of the FACT and MAGIC telescopes. Once the flaring state of the source became evident, it has been observed by the MAGIC telescopes 


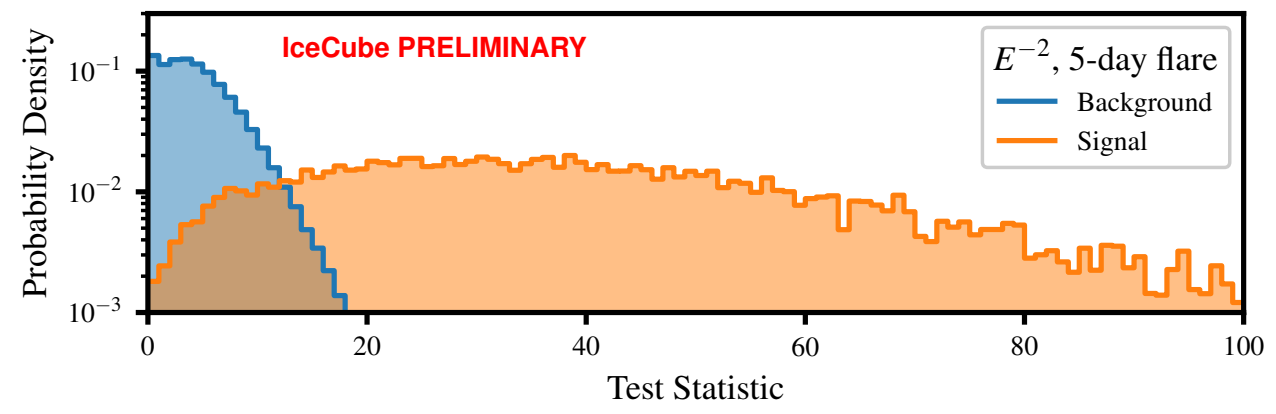

Figure 1: Test statistic $(\Lambda)$ distribution of the time-clustering search. The background distribution (blue) is derived from scrambled, off-time data. The distribution shifts (yellow) as simulated signal events are injected, here drawn with a Poisson mean of 6.02 events in 5 days from an $E^{-2}$ spectrum.

on a regular basis. For 45 out of the 89 nights considered here, the telescopes were able to provide precise measurements of the flux in VHE gamma rays [13]. There are gaps in between the observations, which are largely caused by poor visibility conditions or strong moonlight. Due to the technical design of the FACT camera, it is able to operate in conditions of ambient light and can thus provide measurements for 80 nights [17], adding 36 (MAGIC has one night not included in FACT data) nights of coverage next to the MAGIC observations. Thus, the data from both experiments has been combined to fully utilize their respective strengths and obtain the largest possible coverage of the source behavior over time.

This analysis tests for a direct correlation between the gamma ray and neutrino emission by extending Eqn. 3.1 with an additional PDF in the time domain. In order to construct this time PDF, the telescopes have provided measurements of the integrated gamma ray flux above $750 \mathrm{GeV}$. If on a given night the source has only been observed by one instrument, that measurement is used. On nights where both instruments did provide data, the measured fluxes have been averaged, weighted by their respective errors. As the observations are only possible for a few hours at night-time, the lightcurve has been binned in one day intervals lasting from noon on one day to noon on the next day. This approach strives to balance a conservative extrapolation of the gamma ray flux with a possible time delay between the arrival of neutrinos and gamma rays. In case that no observations were taken on a given night, the surrounding bin is excluded from the analysis. On such nights, no statements on the activity of the source can be made in good conscience, as the strong flaring typically occurs on the timescale of a day. In any case, both a significant flare of neutrinos on an excluded day, as well as a large time delay between the emission of gamma rays and multiple neutrinos would be covered by the two previous analyses, which rely only on the neutrino data.

Figure 2 compares the discovery potential for the three analyses. In general, if single flares in neutrinos with a duration of less than 30 days could be resolved, the time-clustering analysis would be the most sensitive one, whereas for longer time-scales the time-integrated test is more sensitive. The correlation test is very sensitive, if the neutrinos were to follow the gamma ray light curve. Thus, the analyses are complementary as they test different time scales and emission scenarios. 


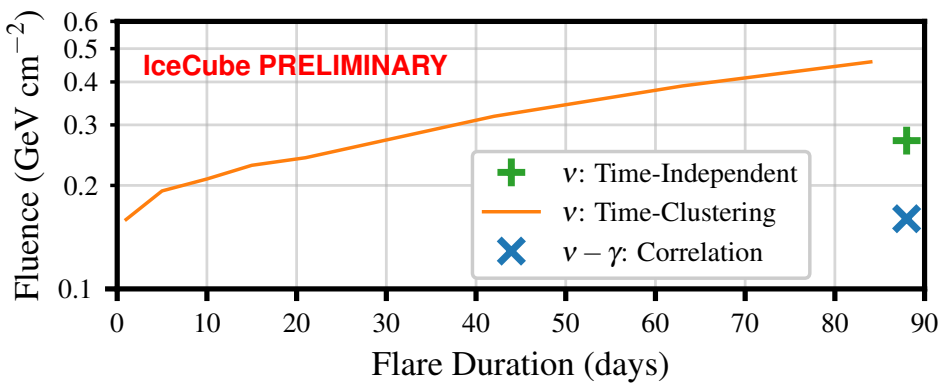

Figure 2: Discovery potential of the three analyses. The time-integrated test is independent of the arrival times of the neutrinos. The sensitivity of the time-clustering method depends on the length of the most-significant flare. The time-correlation test assumes a signal distributed according to the gamma ray lightcurve, yet it is shown in this plot for comparison.

\section{Results}

The analyses have been applied to the 89-day time window from April 29th to July 26th, 2016. Figure 3 shows the location of events, which might contribute to the likelihood analyses due to their proximity to the source. Three events are spatially compatible with the location of 1ES 1959+650 within their $1 \sigma$ error circles, yet they are poorly localized with an uncertainty larger than two degrees.

As the majority of events is well compatible with the background expectation, a test statistic of zero was obtained from the time-integrated test at the position of the source. The right panel of Fig. 3 shows a scan of the test statistic in the vicinity of the source. No spatial clustering is visible near the source.

Figure 4 shows the arrival times and energy proxy of the neutrinos (less than $5 \sigma$ away from the source) as a function of time. The time-clustering test is bound to decide on one most signallike cluster. It selected a time window of 3.3 hours around the most-energetic event in the sample. There are two events in this time window and the fit results yield a signal strength of $n_{s}=2$ and a spectral index of $\gamma=-1.4$. Such an outcome is expected from background with a $p$-value of $37 \%$.
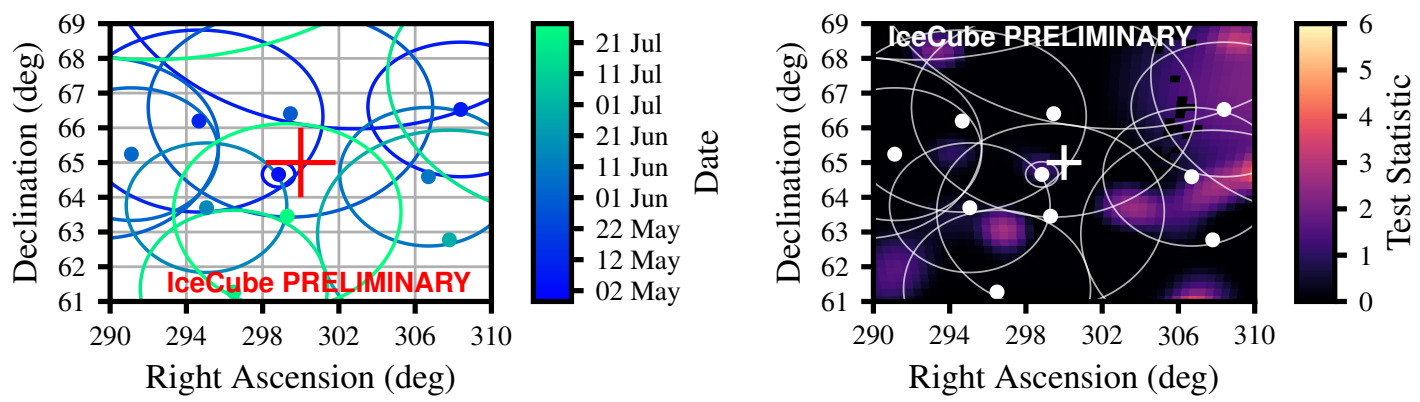

Figure 3: Skymap of the events closer than $2 \sigma$ to the source in equatorial coordinates (J2000). The event locations are shown with a dot; and the circles indicate the $1 \sigma$ uncertainty. Left: The color scale indicates the arrival time. Right: A scan of the test statistic of the time-integrated analysis is shown in the background. 

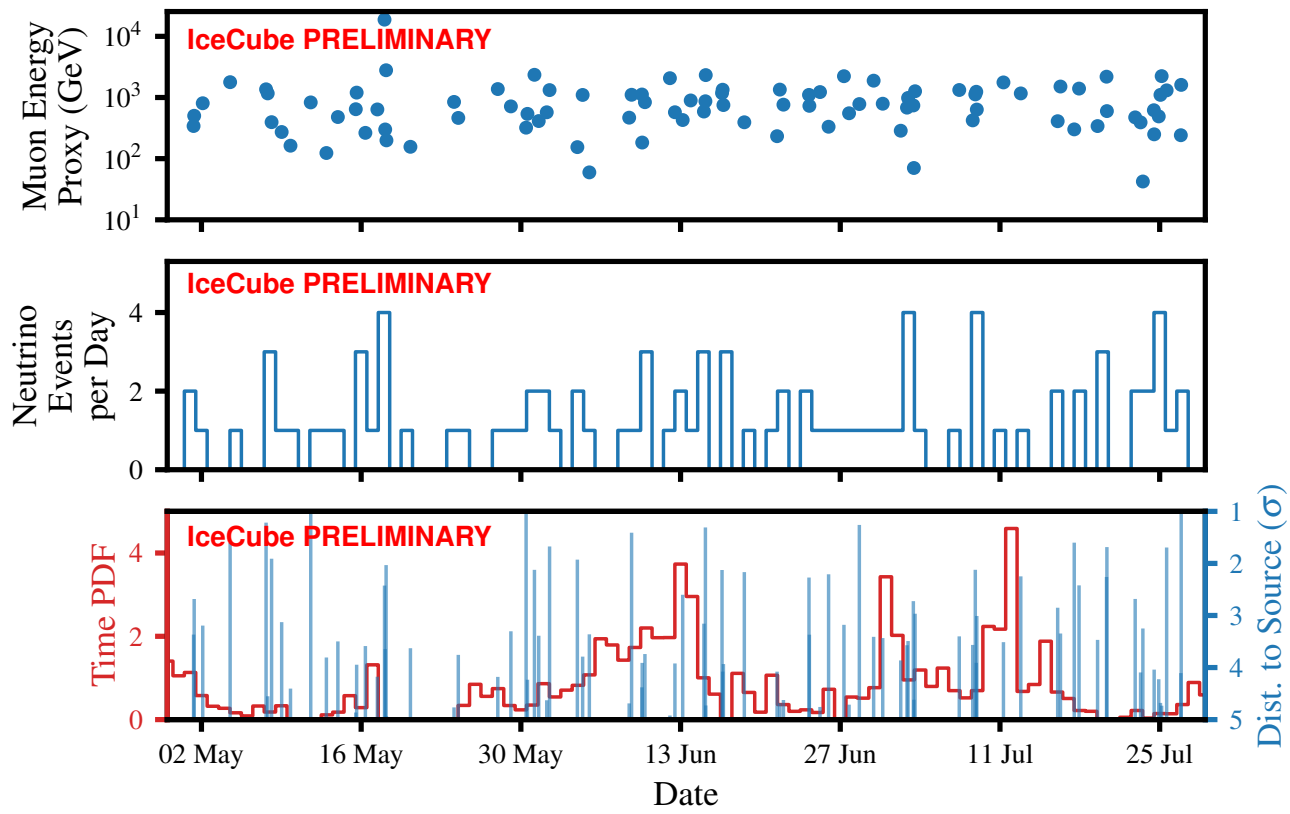

Figure 4: Properties of events compatible with the source location within $5 \sigma$ of their estimated uncertainty, as a function of time. Each event's proxy for the muon energy at the detector [24] is shown in the top plot. The center plot and the red line in the bottom plot show the number of neutrinos and the state in gamma rays (Sec. 3.3), respectively, using the same binning. In the lower plot, the neutrinos' arrival time and distance to the source is overlaid. The most-significant cluster consists of two events observed on May 18th, however it is well compatible with the background expectation.

Additionally, the state of the source in gamma rays is unknown; no observations are available for this particular day.

Finally, due to the absence of signal-like events the final neutrino-gamma correlation test also yields a test statistic of zero and is therefore most-compatible with background.

\section{Summary}

The high-peaked BL Lac object 1ES 1959+650 exhibited major flares in VHE gamma rays in the spring of 2016. A search for neutrinos coincident with the flares has been performed. No significant excess has been observed, neither by integrating over the whole flaring episode, nor by testing for clusters on shorter time scales. Also, no indication for a neutrino flux correlated with the gamma ray flux was found. During the previous episode of strong gamma ray flares from this source in 2002, a neutrino was observed arriving from the location of the source within a few hours of the gamma ray observations [8]. However, back then it was found to be an orphan flare, while for the time considered here, the available X-ray data [25] do not confirm an orphan behavior ${ }^{1}$.

For both the 2002 flare and the recent one, several models attempt to predict possible neutrino fluxes in the light of strong VHE gamma-ray flares: Yet, even with an IceCube-sized detector, a stacking of 40 hours of similar, known orphan flares is required for a detection in the mirror

\footnotetext{
${ }^{1}$ It cannot be excluded during the highest gamma ray emission (June 13th) due to lack of concurrent X-ray data.
} 
model [7, 26]. Similarly, the recent spine-sheath model [27] predicts only 0.04 additional neutrino events on the days of the brightest flares, where the gamma ray flux levels reach 10-15 times that of the low state [11].

Further observations in all wavelengths of this and similar objects are necessary to properly constrain acceleration scenarios. In the context of IceCube's realtime alert program, continued monitoring for potential neutrinos from this source and subsequent, timely alerts to follow-up partner observatories [28] may help to create a larger dataset of simultaneous multi-messenger observations in the future.

\section{References}

[1] F. W. Stecker, O. C. De Jager, and M. H. Salamon, Astrophys. J. 473 (1996) L75-L78.

[2] J. F. Schachter et al., Astrophys. J. 412 (1993) 541-549.

[3] VERITAS Collaboration, J. Holder et al., Astrophys. J. 583 (2003) L9-L12.

[4] T. Nishiyama, ICRC1999 3370.

[5] H. Krawczynski et al., Astrophys. J. 601 (2004) 151-164.

[6] VERITAS Collaboration, M. K. Daniel et al., Astrophys. J. 621 (2005) 181.

[7] M. Böttcher, Astrophys. J. 621 (2005) 176-180. [Erratum: Astrophys. J.641,1233(2006)].

[8] IceCube Collaboration, M. Ackermann et al., ICRC2005 51.

[9] F. Halzen and D. Hooper, Astropart. Phys. 23 (2005) 537-542.

[10] VERITAS Collaboration, E. Aliu et al., Astrophys. J. 775 (2013) 3.

[11] MAGIC Collaboration, K. Satalecka et al., AIP Conf. Proc. 1112 (2009) 223-232.

[12] IceCube Collaboration, M. G. Aartsen et al., Astrophys. J. 835 (2017) 151.

[13] The Astronomer's Telegram 9010, 9139, 9148, 9203, 9239 (2016).

[14] IceCube Collaboration, M. G. Aartsen et al., JINST 12 (2017) P03012.

[15] IceCube Collaboration, M. G. Aartsen et al., Astrophys. J. 833 (2016) 3.

[16] A. Biland et al., JINST 9 (2014) P10012.

[17] FACT Collaboration, D. Dorner, PoS (ICRC2017) 868 (these proceedings).

[18] J. Aleksić et al., Astropart. Phys. 72 (2016) 76-94.

[19] MAGIC Collaboration, M. L. Ahnen et al., arXiv:1704.00906.

[20] MAGIC Collaboration, R. Zanin et al., Braz. J. Phys. ICRC2013 773.

[21] J. Braun et al., Astropart. Phys. 29 (2008) 299-305.

[22] IceCube Collaboration, M. G. Aartsen et al., Astrophys. J. 805 (2015) L5.

[23] R. Abbasi et al., Astrophys. J. 744 (2012) 1.

[24] IceCube Collaboration, M. G. Aartsen et al., JINST 9 (2014) P03009.

[25] M. C. Stroh and A. D. Falcone, Astrophys. J. Suppl. 207 (2013) 28.

[26] A. Reimer, M. Bottcher, and S. Postnikov, Astrophys. J. 630 (2005) 186-190.

[27] C. Righi, F. Tavecchio, and D. Guetta, Astron. Astrophys. 598 (2017) A36.

[28] IceCube, MAGIC, VERITAS Collaboration, M. G. Aartsen et al., JINST 11 (2016) P11009. 